\title{
A density-based topology optimization methodology for thermal energy storage systems
}

\author{
Lundgaard, Christian; Engelbrecht, Kurt; Sigmund, Ole
}

Published in:

Structural and Multidisciplinary Optimization

Link to article, DOI:

$10.1007 / \mathrm{s} 00158-019-02375-8$

Publication date:

2019

Document Version

Peer reviewed version

Link back to DTU Orbit

Citation (APA):

Lundgaard, C., Engelbrecht, K., \& Sigmund, O. (2019). A density-based topology optimization methodology for thermal energy storage systems. Structural and Multidisciplinary Optimization, 60(6), 2189-2204.

https://doi.org/10.1007/s00158-019-02375-8

\section{General rights}

Copyright and moral rights for the publications made accessible in the public portal are retained by the authors and/or other copyright owners and it is a condition of accessing publications that users recognise and abide by the legal requirements associated with these rights.

- Users may download and print one copy of any publication from the public portal for the purpose of private study or research.

- You may not further distribute the material or use it for any profit-making activity or commercial gain

- You may freely distribute the URL identifying the publication in the public portal

If you believe that this document breaches copyright please contact us providing details, and we will remove access to the work immediately and investigate your claim 


\title{
A density-based topology optimization methodology for thermal energy storage systems
}

\author{
Christian Lundgaard $^{\mathrm{a}} \cdot$ Kurt Engelbrecht $^{\mathrm{b}} \cdot$ Ole Sigmund $^{\mathrm{c}}$
}

Received: date / Accepted: date

\begin{abstract}
As many renewable energy resources are prone to an intermittent production of energy and the electric energy demand varies on daily and seasonal time-scales, it is critical to develop technologies which can reduce the residual between the production and the consumption of electric energy. By storing and releasing thermal energy and converting energy between thermal and electric phases, Thermal Energy Storage (TES) systems can be used to reduce this residual.

In this paper, we present a design methodology which can be used to improve the performance of TES systems by distributing two materials with different thermal characteristics in a two dimensional design space. The design methodology is developed with basis in densitybased topology optimization and a transient potential flow model coupled with thermal heat transfer. By solving a sequence of design problems, important model and optimization parameters are identified and the performance of TES systems is increased with $46 \%$ compared to benchmark designs.
\end{abstract}

\section{Introduction}

Motivated by the concerns about anthropogenic climate changes, health impacts of particle pollution and diminishing fossil fuel reserves, the surge for the deploy-

\footnotetext{
a,c Department of Mechanical Engineering

Techinical University of Denmark, Nils Koppels Allé 414, DK2800 Kgs. Lyngby, Denmark

E-mail: chrlund@mek.dtu.dk ${ }^{\mathrm{a}}$, E-mail: sigmund@mek.dtu.dk ${ }^{\mathrm{c}}$

b Department of Energy Conversion and Storage

Techinical University of Denmark, Frederiksborgvej 399, DK4000 Roskilde, Denmark

E-mail: kuen@dtu.dk
}

ment of renewable energy technologies have intensified considerably within the last decades.

The harvesting of wind and solar energy are exposed to a large amount of scientific and commercial attention, as these technologies are considered some of the most promising entrants in the green energy changeover (Lund, 2007, Wang, 2010). As the harvesting of wind and solar energy relies on the fluctuating and unpredictable nature of weather systems, a focal challenge of these technologies is to minimize the mismatch between the supply and the demand of electric power, the so-called residual load (Laughlin, 2017).

TES systems can be used to store a surplus of energy generated from intermittent energy production technologies such as wind turbines or solar panels (Agyenim et al., 2010, Kearney et al., 2003). By storing and releasing thermal energy and converting energy between thermal and electric phases, Thermal Energy Storage (TES) systems serve as energy batteries and can therefore be used to reduce the residual between the consumption and production of electric power.

Compared to energy storage systems such as chemical batteries, TES systems have several advantages for which reason they have been seen in several applications such as cooling and refrigeration (Chen et al., 2009), solar power plants (Gil et al., 2010), solar cooking (Muthusivagami et al., 2010) and seasonal energy storage (Novo et al., 2010). For a detailed introduction to TES systems see the work of McTigue (2016) and the references therein.

A packed-bed TES systems can with reference to Figs. 1a and 1b be decomposed into four major parts: (1) porous thermal storage mixtures composed of e.g. gravel/rocks with relatively high permeability and sand with relatively low permeability. These material phases are in the figure colored with and and com- 


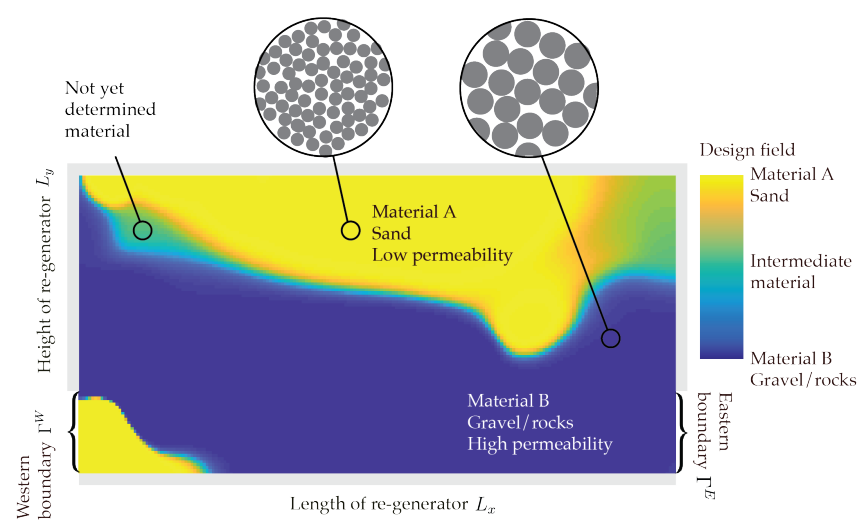

(a) A sketch of a TES system and the conceptual background for the design problem. The aim of the design problem is to spatially distribute two different materials, Material A (sand) and Material B (gravel/rocks) in a two dimensional design space in order to optimize the performance of the TES system.

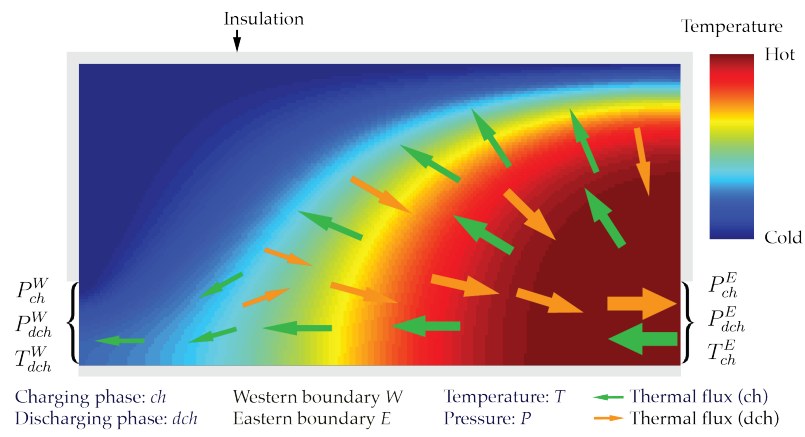

(b) Thermal energy is absorbed in the storage matrix during the charging phase and extracted from the storage matrix during the discharging phase. The flow is driven by pressure differences between the eastern and western boundaries. The temperature field is plotted at a suitable time during the charging cycle for a storage matrix consisting exclusively of gravel/rocks.

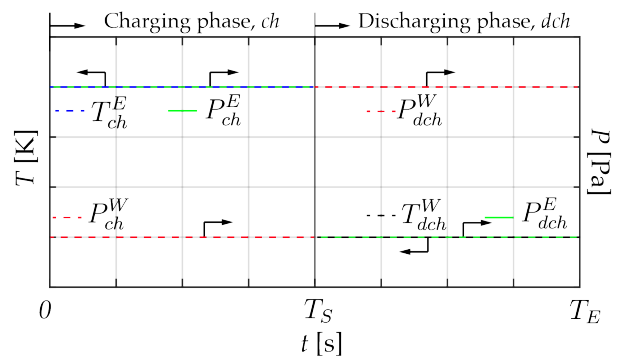

(c) A schematic representation of the pressure and temperature boundary conditions during the charging and discharging phases. The charging and discharging phases, $c h$ and $d_{c h}$, are for the time $t$ defined by the intervals $t \in\left[0 ; T_{S}\right]$ and $\left.\left.t \in\right] T_{S} ; T_{E}\right]$, respectively. The aim of the optimization problem is to maximize the heat flux though the eastern surface, $\Gamma^{E}$, during the discharge phase.

Fig. 1: Schematics of (a) a TES system and the design problem concept, (b) a conceptual example of the governing physics and (c) a schematic of the boundary conditions solved using the design methodology. bined referred to as the storage matrix. (2) a heat transfer fluid which is used to absorb and extract thermal energy in the porous storage matrix. (3) a compartment which is insulated and pressurised to drive the heat transfer fluid and reduce the thermal losses to the ambient. (4) boundaries where an inlet and an outlet can be positioned such that the heat transfer fluid can enter and exit the storage matrix. These boundaries are referred to as the eastern boundary and the western boundary, $\Gamma^{E}$ and $\Gamma^{W}$, respectively.

As TES systems absorb and extract thermal energy in and from the storage matrix, the temperature is the primary physical field of interest, see Fig. 1b. A TES system is operating in cycles consisting of two phases: (1) a charging phase where thermal energy is absorbed in the storage matrix and (2) a discharging phase where thermal energy is extracted from the storage matrix. As the path of propagation of thermal energy is irreversible, the temperature response of TES systems is required to be modeled time dependently.

To demonstrate the propagation of thermal energy through the storage mixture, we have in Fig. 1b plotted the temperature field during the charging phase of a TES system consisting exclusive of gravel/rocks. The arrows on the figure illustrate that the temperature is propagating from the eastern boundary towards the western boundary during the charging phase and from the western boundary towards the eastern boundary during the discharging phase.

With reference to Fig. 1c, the charging and discharging phases are conducted via an interplay between the temperature and pressure of the heat transfer fluid. Thermal energy is absorbed in the storage matrix during the charging phase, as hot fluid propagates from the eastern boundary towards the western boundary. The flow is driven by the pressure difference between the eastern and western boundary. By reversing the flow during the discharge phase, the thermal energy is extracted from the storage matrix, as the fluid can escape through the eastern boundary.

The performance of TES systems can be quantified by two measures: (1) the total amount of thermal energy which can be absorbed in the storage matrix and (2) the time it takes to absorb and extract the thermal energy from the storage matrix. As the sand and gravel/rock material phases in Fig. 1a have considerably different physical properties, the hypothesis of the present study is the following: The performance of TES systems can be improved considerably by systematically distributing sand and gravel/rocks in the storage matrix. As the physical modeling and the performance of TES systems are dependent on a large number of parameters such as time, material parameters, model dimensions, bound- 
ary conditions, the architecture of the charging and discharging phases and the choice of objective function, we are convinced that the "optimal" topology of the storage matrix is complex. Due to the complexity of the design problem, we believe that a systematic material distribution approach is best suited for determining the topology of the sand and gravel/rocks in the storage matrix. With point of departure in this motivation and hypothesis, we have derived, implemented and presented a density-based topology optimization approach for TES systems in the present study.

Topology optimization originates from solid mechanics (Bendsøe and Sigmund, 2003, Bendsøe and Kikuchi, 1988) but has been applied in many different types of multi-physical problems in resent decades such as natural convection (Alexandersen et al., 2014), reacting flows (Okkels and Bruus, 2007), thermoelectric energy conversion (Lundgaard and Sigmund, 2019), fluid-structureinteraction (Yoon, 2010) and many more. We suggest the interested reader to consult the work of Deaton and Grandhi (2014) who provide a comprehensive literature study for the multidisciplinary scientific work in topology optimization. Transient topology optimization problems have been studied for flows (Deng et al., 2011, Nørgaard et al., 2016), crass-worthiness (Pedersen, 2004), wave propagation problems (Dahl et al., 2008), thermally actuated compliant mechanisms ( $\mathrm{Li}$ et al., 2004) and many more. The framework presented in the present paper is transient and multi-physical and is therefore related to the works listed above.

A density-based topology optimization methodology for steady-state potential flows coupled with thermal transport was presented in the work of Zhao et al. (2018). The design methodology presented in the present paper is related to this work, but is conceptually very different with respect to design problems and modeling approaches. Where Zhao and coworkers aimed at developing a computationally efficient alternative to solve the full turbulent thermally coupled Navier-Stokes equations, the methodology presented here is concerned with transient design problems in porous media and effective thermal energy storage systems.

\section{Physical model}

With reference to Fig. 1a the material phases in the storage matrix are porous. As the inertia forces of fluid flows in porous medias are negligible, a potential flow model is used to model the fluid flow. Potential flow models are equivalent to Darcy's law for flow in porous medias (Whitaker, 1986). The potential flow model is coupled to a heat transport model via the contribution from two separately identified heat transfer types: conductive heat transfer and convective heat transfer.

The density or compressibility of the fluid are assumed constant or negligible, respectively. As the lengthscales of the storage mixtures are small and the transfer of thermal energy between the heat transfer fluid and the storage mixtures is effective, it is reasonable to assume that the local temperature of a small region of the re-generator is constant (Laughlin, 2017). As there are no temperature difference between the storage matrix and the heat transfer fluid for a very small and local region of the re-generator, the temperature field can be adequately modeled with a single set of temperature equations, the so-called volume averaging technique (Whitaker, 1986). By further limiting the physical modeling to linear/constant material parameters, the continuity of thermal energy and momentum in the fluid are in an arbitrary domain, $\Omega$, given by:

$$
\begin{array}{r}
\rho \nabla \cdot \mathbf{u}=F \quad \text { in } \quad \Omega \\
\rho c_{p} \frac{\partial T}{\partial t}+\rho c_{p} \mathbf{u} \cdot \nabla T=k \nabla^{2} T+P \quad \text { in } \quad \Omega
\end{array}
$$

where $\rho$ the mass density $\left[\mathrm{kg} / \mathrm{m}^{3}\right], \nabla$ is the partial derivative with respect to spatial directions $x$ and $y$ $[1 / \mathrm{m}], \mathbf{u}$ is the velocity vector $[\mathrm{m} / \mathrm{s}], F$ is the volumetric body force $[\mathrm{N}], c_{p}$ specific heat capacity $[\mathrm{J} / \mathrm{kg} \cdot \mathrm{K}], t$ is the time $[\mathrm{s}], T$ is the temperature $[\mathrm{K}], k$ is the thermal conductivity $[\mathrm{W} / \mathrm{K} \cdot \mathrm{m}]$ and $P$ is the internal heat generation $\left[\mathrm{W} / \mathrm{m}^{3}\right]$. The boundary conditions of Eq. (1) are given by:

Fixed pressure $P=c_{1}$

Fixed temperature $T=c_{2}$

Stress free surface $\mathbf{n} \cdot \mathbf{P}=0$

Thermal insulation $\mathbf{n} \cdot \mathbf{Q}=0$

where $\mathbf{n}$ is a vector normal to the boundary at which is boundary condition is imposed, $c_{1}$ and $c_{2}$ are numbers larger or equal 0 .

In Eq. (1), the fluid pressure and fluid velocity are related via the following equation:

$\mathbf{u}=-\frac{\kappa}{\mu} \nabla p$

where $\kappa$ is the permeability $\left[\mathrm{m}^{2}\right], \mu$ the dynamic viscosity $[\mathrm{Pa} \cdot \mathrm{s}]$ and $p$ the pressure $[\mathrm{Pa}]$.

The heat flux, $\mathbf{Q}$, is simply the sum of the convective heat transfer and the conductive heat transfer:

$\mathbf{Q}=\rho c_{p} \mathbf{u} \cdot \nabla T-k \nabla^{2} T$

As a considerable amount of abbreviations and variables are used throughout the paper, we have for readability purposes listed the most used ones in Tab. 1. 
Table 1: List of important variables used throughout the paper.

\begin{tabular}{|c|c|}
\hline Variable & Description \\
\hline$\Gamma^{E}$ & Eastern boundary \\
\hline$\Gamma^{W}$ & Western boundary \\
\hline$T_{\sqcap}^{\circ}$ & Temperature boundary condition \\
\hline$P_{\square}^{0}$ & Pressure boundary condition \\
\hline$O=E$ & Eastern boundary, $\Gamma^{E}$ \\
\hline $0=W$ & Western boundary, $\Gamma^{W}$ \\
\hline$\square=c h$ & charging phase: $t \in\left[T_{0} ; T_{S}\right]$ \\
\hline$\square=d c h$ & 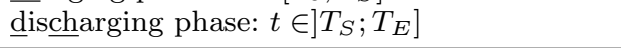 \\
\hline$T_{0}$ & Start of charging phase $[\mathrm{s}]$ \\
\hline$T_{S}$ & End of charging phase $[\mathrm{s}]$ \\
\hline$T_{S}$ & Start of discharging phase $[\mathrm{s}]$ \\
\hline$T_{E}$ & End of discharging phase $[\mathrm{s}]$ \\
\hline $\mathbf{P}$ & The pressure field $[\mathrm{Pa}]$ \\
\hline $\mathbf{T}$ & The temperature field $[\mathrm{K}]$ \\
\hline $\mathbf{S}$ & $\mathbf{S}=\{\mathbf{P}, \mathbf{T}\}^{T}$ \\
\hline $\mathrm{t}$ & Time $[\mathrm{s}]$ \\
\hline$\dot{\mathbf{S}}$ & $\dot{\mathbf{S}}=\partial \mathbf{S} / \partial t$ \\
\hline & The velocity field $[\mathrm{m} / \mathrm{s}]$ \\
\hline $\mathbf{Q}_{x}, \mathbf{Q}_{y}$ & The thermal heat flux field \\
\hline $\mathrm{x}$ & $\begin{array}{l}\text { in } x \text { and } y, \text { respectively } \\
\text { The design field [-] }\end{array}$ \\
\hline$f_{Q}$ & Heat flux objective function \\
\hline$\Omega$ & Design and computational domain \\
\hline$L_{x}$ & Length of $\Omega$ in $x$ \\
\hline$L_{y}$ & Length of $\Omega$ in $y$ \\
\hline $\mathbb{N}$ & Index set for finite elements in $\Omega$ \\
\hline $\mathbb{M}$ & Index set for time steps in $\left.t \in] T_{S} ; T_{E}\right]$ \\
\hline $\mathbb{0}$ & Index set for $x$-directional heat fluxes on $\Gamma^{E}$ \\
\hline
\end{tabular}

\section{Finite element formulation}

The topology optimization approach takes basis in the idea of spatially distributing two different material phases (Material A or Material B) in a two dimensional design space, $\Omega$, in order to optimize for a specified performance measure. To cast the equations in Eqs. (1a)-(1b) on a form which is suitable for density-based topology optimization, we introduce a design variable field, $0 \leq x \leq 1$, such that the material parameters become functions of the design field, i.e. $\rho=\rho(x), c_{p}=c_{p}(x)$, $k=k(x), \kappa=\kappa(x)$ and $\mu=\mu(x)$. The design variable field can now be used to control whether a part of the design domain behaves physically like Material A, $x=0$; Material $\mathrm{B}, x=1$; or an intermediate state between Material A and Material B, $0<x<1$.

The discretized finite element equations can now be obtained by multiplying the strong forms of the equations in Eq. (1a)-(1b) with suitable test functions; integrating over the domain; performing integration by parts of higher dimensions on relevant terms; and introducing the design field dependent interpolation functions (Cook et al., 2007).

The most general form of the finite element residual equation is given by:

$$
\begin{aligned}
\mathbf{R}= & \mathbf{M}(\mathbf{x}, \mathbf{S}(\mathbf{x}, t)) \dot{\mathbf{S}}(\mathbf{x}, t) \\
& +\mathbf{K}(\mathbf{x}, \mathbf{S}(\mathbf{x}, t)) \mathbf{S}(\mathbf{x}, t)-\mathbf{F}(\mathbf{S}(\mathbf{x}, t))=\mathbf{0}
\end{aligned}
$$

where $\mathbf{R}$ is the residual vector, $\mathbf{M}$ is the mass matrix, $\mathbf{x}$ is the design variable field, $t$ is the time, $\mathbf{S}$ is the state field vector, $\dot{\mathbf{S}}$ is the time derivative of the state variable vector, $\mathbf{K}$ is the system matrix, and $\mathbf{F}$ is the load vector. The state field vector and the time-derivative of the state field vector are given by:

$\mathbf{S}=\left\{\begin{array}{l}\mathbf{P} \\ \mathbf{T}\end{array}\right\} \quad \dot{\mathbf{S}}=\left\{\begin{array}{c}\frac{\partial \mathbf{P}}{\partial t} \\ \frac{\partial \mathbf{T}}{\partial t}\end{array}\right\}$

where $\mathbf{P}$ is the pressure and $\mathbf{T}$ is the temperature. The discretized system of equations in Eq. (5) can be written as:

$$
\begin{aligned}
\left\{\begin{array}{l}
\mathbf{R}_{P P} \\
\mathbf{R}_{T T}
\end{array}\right\} & =\left[\begin{array}{ll}
\mathbf{0} & \mathbf{0} \\
\mathbf{0} & \mathbf{M}_{T T}
\end{array}\right]\left\{\begin{array}{l}
\dot{\mathbf{P}} \\
\dot{\mathbf{T}}
\end{array}\right\} \\
& -\left[\begin{array}{cc}
\mathbf{K}_{P P} \\
\mathbf{0} & \mathbf{K}_{T T}+\mathbf{C}_{T T}+\mathbf{L}_{T T}
\end{array}\right]\left\{\begin{array}{l}
\mathbf{P} \\
\mathbf{T}
\end{array}\right\} \\
& -\left\{\begin{array}{l}
\mathbf{F}_{P P} \\
\mathbf{F}_{T T}
\end{array}\right\}=\mathbf{0}
\end{aligned}
$$

where the element system matrices are given by:

$$
\begin{aligned}
\mathbf{M}_{T T}(x) & =\sum_{i \in \mathbb{N}} \int_{\Omega^{e}} \rho(x) c_{p}(x) \mathbf{N}^{T} \mathbf{N} \mathrm{d} V \\
\mathbf{K}_{P P}(x) & =\sum_{i \in \mathbb{N}} \int_{\Omega^{e}} \frac{\kappa(x)}{\mu} \mathbf{B}^{T} \mathbf{B} \mathrm{d} V \\
\mathbf{K}_{T T}(x) & =\sum_{i \in \mathbb{N}} \int_{\Omega^{e}} k(x) \mathbf{B}^{T} \mathbf{B} \mathrm{d} V \\
\mathbf{C}_{T T}(x, P) & =\sum_{i \in \mathbb{N}} \int_{\Omega^{e}} \rho(x) c_{p}(x) \mathbf{N}^{T} \mathbf{u}^{T} \mathbf{B} \mathrm{d} V \\
\mathbf{L}_{T T}(x, P) & =\sum_{i \in \mathbb{N}} \int_{\Omega^{e}} \rho(x) c_{p}(x) \hat{\mathbf{N}} \mathbf{u}^{T} \mathbf{B} \mathrm{d} V \\
\mathbf{F}_{P P} & =\sum_{i \in \mathbb{N}} \int_{\Gamma^{e}} \mathbf{N}^{T} \mathbf{n} \cdot \mathbf{u} \mathrm{d} V \\
\mathbf{F}_{T T} & =\sum_{i \in \mathbb{N}} \int_{\Gamma^{e}} \mathbf{N}^{T} Q \mathrm{~d} V
\end{aligned}
$$

$\sum_{i \in \mathbb{N}}$ denotes a standard finite element assembly procedure for element $i$ over the index set of elements to be assembled, $\mathbb{N} ; \mathbf{N}$ is the matrix of element shape functions and $\mathbf{B}$ is the derivative of $\mathbf{N}$ namely, $\mathbf{B}=\nabla_{x} \mathbf{N}$, where $\nabla_{x}$ denotes the differentiation operation with respect 
to the spatial coordinates. Lower case letters generally denote element stiffness matrices and vectors and capital letters generally denote global stiffness matrices and vectors. The naming of the element matrices in Eqs. (8) is the following: $\mathbf{M}_{T T}$ is the thermal mass matrix, $\mathbf{K}_{P P}$ is the pressure stiffness matrix; $\mathbf{K}_{T T}$ is the thermal conduction stiffness matrix; $\mathbf{C}_{T T}$ is the thermal convection stiffness matrix; $\mathbf{L}_{T T}$ is the thermal stabilization matrix; $\mathbf{F}_{P P}$ is the pressure loads and $\mathbf{F}_{T T}$ is the thermal loads. The shape function, $\hat{\mathbf{N}}$, for the thermal stabilization stiffness matrix $\mathbf{L}_{T T}$ in Eq. (8e) is given by:

$\hat{\mathbf{N}}=\frac{h^{e}}{2}\left(\frac{\mathbf{u}}{\|\mathbf{u}\|_{2}}\right)^{T} \mathbf{B}$

where $\|\mathbf{u}\|_{2}=\sqrt{\mathbf{u}^{T} \mathbf{u}}$.

The element velocity can be computed from the pressure by the following relationship:

$\mathbf{U}=\sum_{i \in \mathbb{N}} \int_{\Gamma^{e}} \frac{\kappa}{\mu} \mathbf{B}^{T} \mathbf{p}^{e} \mathrm{~d} V$

where $\mathbf{p}^{e}$ is the pressure in the element which is evaluated. The element heat flux can now be computed by:

$\mathbf{Q}=\sum_{i \in \mathbb{N}} \rho c_{p} \mathbf{u} \mathbf{N}^{T} \mathbf{t}^{e}+k \mathbf{B} \mathbf{t}^{e}$

where $\mathbf{t}^{e}$ is the temperature in the element which is evaluated. Please notice that the contribution from the stabilization is not included in the heat flux in Eq. 11. The contribution from the stabilization on the heat flux is given by $\rho c_{p} \mathbf{u} \hat{\mathbf{N}}^{T} \mathbf{t}^{e}$.

The material parameters are dependent on the design field, $x$, and are interpolated between the material phases by the following interpolation functions (Borrvall and Petersson, 2003):

$\square(x)=\square^{A}+\frac{\left(\square^{B}-\square^{A}\right)(1-x)(1+\bigcirc)}{1-x+\bigcirc}$

$\square$ and $\bigcirc$ can take the entries $\square \in\left\{\rho, c_{p}, \kappa, k\right\}$ and $\bigcirc \in$ $\left\{p_{\rho}, p_{c_{p}}, p_{\kappa}, p_{k}\right\}$ and denote the material parameters for Material A and B in Fig. 1 and the interpolation function penalization parameters, respectively. The symbols ${ }^{A}$ and ${ }^{B}$ refer to the material parameters for Material A and $B$, respectively. Intermediate design variables in Eq. (12) may not be physically meaningful, however if design variables take values in the extremes of the set, i.e. $x \in\{0,1\}$, the design variables are physical meaningful.

The derivatives of the residual equation in Eq. (5) with respect to state variables are required and given by:

$$
\begin{aligned}
& \frac{\partial \mathbf{R}}{\partial \mathbf{S}}=\frac{\partial \mathbf{M}}{\partial \mathbf{S}} \dot{\mathbf{S}}+\mathbf{M} \frac{\partial \dot{\mathbf{S}}}{\partial \mathbf{S}}+\frac{\partial \mathbf{K}}{\partial \mathbf{S}} \mathbf{S}+\mathbf{K} \frac{\partial \mathbf{S}}{\partial \mathbf{S}}-\frac{\partial \mathbf{F}}{\partial \mathbf{S}} \\
& \frac{\partial \mathbf{R}}{\partial \dot{\mathbf{S}}}=\frac{\partial \mathbf{M}}{\partial \dot{\mathbf{S}}} \dot{\mathbf{S}}+\mathbf{M} \frac{\partial \dot{\mathbf{S}}}{\partial \dot{\mathbf{S}}}+\frac{\partial \mathbf{K}}{\partial \dot{\mathbf{S}}} \mathbf{S}+\mathbf{K} \frac{\partial \mathbf{S}}{\partial \dot{\mathbf{S}}}-\frac{\partial \mathbf{F}}{\partial \dot{\mathbf{S}}}
\end{aligned}
$$

$\partial \square / \partial \bigcirc$ denotes the derivative of $\square$ with respect to $\bigcirc$. Eq. (13) reduces to:

$$
\begin{aligned}
& \frac{\partial \mathbf{R}}{\partial \mathbf{S}}=\frac{\partial \mathbf{M}}{\partial \mathbf{S}} \dot{\mathbf{S}}+\frac{\partial \mathbf{K}}{\partial \mathbf{S}} \mathbf{S}+\mathbf{K}-\frac{\partial \mathbf{F}}{\partial \mathbf{S}} \\
& \frac{\partial \mathbf{R}}{\partial \dot{\mathbf{S}}}=\mathbf{M}
\end{aligned}
$$

which in details are given by:

$$
\begin{aligned}
\frac{\partial \mathbf{R}}{\partial \mathbf{S}}= & {\left[\begin{array}{c}
\mathbf{0} \frac{\partial \mathbf{M}_{P P}}{\partial \mathbf{T}} \cdot \dot{\mathbf{P}} \\
\mathbf{0} \frac{\partial \mathbf{M}_{T T}}{\partial \mathbf{T}} \cdot \dot{\mathbf{T}}
\end{array}\right]+} \\
\mathbf{0} & {\left[\begin{array}{c}
\frac{\partial \mathbf{K}_{P P}}{\partial \mathbf{T}} \cdot \mathbf{P} \\
\frac{\partial \mathbf{P}}{T T} \\
+\mathbf{T}+\frac{\partial \mathbf{L}_{T T}}{\partial \mathbf{P}} \cdot \mathbf{T} \frac{\partial \mathbf{K}_{T T}}{\partial \mathbf{T}} \cdot \mathbf{T}+\frac{\partial \mathbf{C}_{T T}}{\partial \mathbf{T}} \cdot \mathbf{T}+\frac{\partial \mathbf{L}_{T T}}{\partial \mathbf{T}} \cdot \mathbf{T}
\end{array}\right] } \\
& +\mathbf{K}-\left[\begin{array}{cc}
\mathbf{0} \frac{\partial \mathbf{F}}{\partial \mathbf{T}} \\
\mathbf{0} & \mathbf{0}
\end{array}\right]
\end{aligned}
$$

where the dot product notation, [·], between a matrix and a vector denotes the sum over the nearest indices in tensor notation. If $\mathbf{A}$ is a matrix and $\mathbf{b}$ is a vector, then $\mathbf{A} \cdot \mathbf{b}$ is equivalent to $A_{i j} b_{j}$ in tensor notation.

The derivative of the residual vector with respect to the state field in Eqs. (15) is achieved by assembling each system matrix independently, e.g.:

$$
\frac{\partial \mathbf{M}_{P P}}{\partial \mathbf{T}} \cdot \dot{\mathbf{P}}=\sum_{i \in \mathbb{N}} \frac{\partial \mathbf{m}_{P P}^{e}}{\partial \mathbf{t}^{e}} \cdot \dot{\mathbf{p}}^{e}, \quad \ldots
$$

where ... denotes the remaining terms in Eq. (15).

The governing partial differential equations in Eqs. (1) are time dependent for which reason a time stepping scheme is required to solve the transient response of the heat transfer problem. An implicit second order backward differentiation formula scheme is used:

$\left(\mathbf{M}+\Delta t \mathbf{K}_{n}\right) \mathbf{S}_{n+1}=\Delta t \mathbf{F}+\mathbf{M} \mathbf{S}_{n}$

where $n[-]$ denotes the time step and $\Delta t[\mathrm{~s}]$ denotes the time step length.

As the pressure is dependent on the temperature but the pressure is independent of the temperature, the governing equations in Eqs. (1) are weakly coupled. The unified architecture of the system of equations in Eqs. (8) is computationally inefficient compared to the corresponding separated architecture where the temperature 
and pressure equations are solved separately. However, the computationally efficient form can easily be obtained by rewriting Eq. (8). We have decided to present the design methodology in the unified architecture, as this form straight forwardly supports extensions to more advanced physical modeling such as temperature dependent material parameters and compressible fluid.

\section{Topology optimization}

The optimization problem solved in the present study can in general form be written as:

$\min _{x} f$

s.t. $\mathbf{R}(\tilde{\overline{\mathbf{x}}}, \mathbf{S})=\mathbf{0}$

$\mathbf{0} \leq \mathbf{x} \leq \mathbf{1} \quad \forall \mathbf{x} \in \Omega_{D}$

where $f$ is the objective function, $\mathbf{R}$ is the residual vector, $\tilde{\overline{\mathbf{x}}}$ is the filtered and projected design variables and $\mathbf{x}$ is the design variables.

\subsection{Adjoint sensitivity analysis}

The gradients of the objective function, $f$, with respect to the design variable field, $x$, are required in order to solve the optimization problem in Eq. (18). With $L$ being the general Lagrangian functional, the sensitivities, $\mathrm{d} L / \mathrm{d} \mathbf{x}$, are computed by the discrete adjoint approach (see Bendsøe and Sigmund (2003), Dahl et al. (2008) and the references therein).

The adjoint sensitivity analysis is carried out in a differentiate-then-discretize approach though this approach may provide inconsistant sensitivities. As the design problems have many degrees of freedom, we argue that the error is neglectiable as discussed in the work of Jensen et al. (2014).

The objective function, $f$, in Eq. (18) is given by:

$f=\int_{T_{S}}^{T_{E}} G(\mathbf{x}, \mathbf{S}(\mathbf{x}, t), \dot{\mathbf{S}}(\mathbf{x}, t)) \mathrm{d} t$

where $G$ is a differential function and in this work called the inner objective function. The limits of the integral, $T_{S}$ and $T_{E}$, specify the beginning and the end of the time interval at which the objective function is optimized (the discharging phase). The objective function in Eq. (19) is augmented with the product of the Lagrange multipliers, $\boldsymbol{\lambda}(t)$, and the residual, $\mathbf{R}$ :

$f=\int_{T_{S}}^{T_{E}}\left[G+\boldsymbol{\lambda}^{T}(\mathbf{R})\right] \mathrm{d} t$ where $\square^{T}$ denotes the matrix or vector transpose operation. The derivative of the objective function with respect to the design field is given by:

$$
\begin{aligned}
\frac{\mathrm{d} f}{\mathrm{~d} \mathbf{x}}= & \int_{T_{S}}^{T_{E}}\left[\frac{\partial G}{\partial \mathbf{x}}+\frac{\partial G}{\partial \mathbf{S}} \frac{\partial \mathbf{S}}{\partial \mathbf{x}}+\right. \\
& \left.+\boldsymbol{\lambda}^{T}\left(\frac{\partial \mathbf{R}}{\partial \mathbf{x}}+\frac{\partial \mathbf{R}}{\partial \mathbf{S}} \frac{\partial \mathbf{S}}{\partial \mathbf{x}}+\frac{\partial \mathbf{R}}{\partial \dot{\mathbf{S}}} \frac{\partial \dot{\mathbf{S}}}{\partial \mathbf{x}}\right)\right] \mathrm{d} t
\end{aligned}
$$

where $\frac{d \square}{d \square}$ denotes the total derivative and $\frac{\partial \square}{\partial \square}$ denotes the partial derivative.

By rearranging, performing integration by parts on relevant terms and collecting all terms with $\partial \mathbf{S} / \partial \mathbf{x}$, Eq. (21) can be rewritten to:

$$
\begin{aligned}
\frac{\mathrm{d} f}{\mathrm{~d} \mathbf{x}}=\int_{T_{S}}^{T_{E}} & {\left[\frac{\partial G}{\partial \mathbf{x}}+\boldsymbol{\lambda}^{T} \frac{\partial \mathbf{R}}{\partial \mathbf{x}}\right] \mathrm{d} t+} \\
\int_{T_{S}}^{T_{E}} & {\left[\frac{\partial G}{\partial \mathbf{S}}+\boldsymbol{\lambda}^{T} \frac{\partial \mathbf{R}}{\partial \mathbf{S}}-\dot{\boldsymbol{\lambda}}^{T} \frac{\partial \mathbf{R}}{\partial \dot{\mathbf{S}}}\right] \frac{\partial \mathbf{S}}{\partial \mathbf{x}} \mathrm{d} t } \\
+ & {\left[\boldsymbol{\lambda}^{T} \frac{\partial \mathbf{R}}{\partial \dot{\mathbf{S}}} \frac{\partial \mathbf{S}}{\partial \mathbf{x}}\right]_{0}^{T} }
\end{aligned}
$$

After discretization of the time integrals, Eq. (22) can be written as a time-dependent adjoint problem. The Lagrangian multipliers, $\boldsymbol{\lambda}(t)$ and $\dot{\boldsymbol{\lambda}}(t)$, are required in each time-step to ensure that the terms containing $\partial \mathbf{S} / \partial \mathbf{x}$ in Eq. 22 are zero. This results in an adjoint problem given by:

$$
\frac{\partial G}{\partial \mathbf{S}}=\left[\boldsymbol{\lambda}^{T} \frac{\partial \mathbf{R}}{\partial \mathbf{S}}-\dot{\boldsymbol{\lambda}}^{T} \frac{\partial \mathbf{R}}{\partial \dot{\mathbf{S}}}\right]
$$

Assuming that $\boldsymbol{\lambda}$ and $\dot{\boldsymbol{\lambda}}$ in Eq. (23) are known, the sensitivities in Eq. (22) reduce to:

$$
\frac{\mathrm{d} f}{\mathrm{~d} \mathbf{x}}=\sum_{j \in \mathbb{M}}\left[\frac{\partial G}{\partial \mathbf{x}}-(\boldsymbol{\lambda})^{T} \frac{\partial \mathbf{R}}{\partial \mathbf{x}}\right] \mathrm{d} t
$$

where $j$ is the $j$ 'th time-step in the index set containing the time-steps enclosed by the time integral limits, $\mathbb{M}$. Or explained in an other way: $\sum_{j \in \mathbb{M}}$ is simply the discrete way of writing the time-integral, $\int_{T_{S}}^{T_{E}} \mathrm{~d} t$.

The sums with the index set in Eq. (24) can be exchanged by the vector $\mathbf{L}_{\mathbb{M}}^{T}$ such that the equation can be written in what we call the implementation form:

$\frac{\mathrm{d} f}{\mathrm{~d} \mathbf{x}}=\mathbf{L}_{\mathbb{M}}^{T}\left[\frac{\partial G}{\partial \mathbf{x}}-(\boldsymbol{\lambda})^{T} \frac{\partial \mathbf{R}}{\partial \mathbf{x}}\right]$

$\mathbf{L}_{\mathbb{M}}^{T}$ is a vector with length $N$ consisting of zeros except for the entries of the time steps enclosed by $\left.t \in] T_{S} ; T_{E}\right]$ which have the value $1 / M$. The variables, $M$ and $N$, are the number of time steps enclosed by $\left.t \in] T_{S} ; T_{E}\right]$ and $t \in\left[0, T_{E}\right]$, respectively. As functions cast in the implementation form simplify the implementation complexity 
in a scripting languages such as Matlab og Python, we prefer to provide implementation details in this form.

Aside from the variations of the inner objective function, we still require the derivative of the residual vector with respect to the design variables, $\partial \mathbf{R} / \partial \mathbf{x}$, to know all terms in Eq. (25). This term is given by:

$$
\begin{aligned}
\frac{\partial \mathbf{R}}{\partial \mathbf{x}} & =\left\{\begin{array}{l}
\frac{\partial \mathbf{M}_{P P}}{\partial \mathbf{x}} \cdot \dot{\mathbf{P}} \\
\frac{\partial \mathbf{M}_{T T}}{\partial \mathbf{x}} \cdot \dot{\mathbf{T}}
\end{array}\right\} \\
+ & \left\{\begin{array}{c}
\frac{\partial \mathbf{K}_{P P}}{\partial \mathbf{x}} \cdot \mathbf{P} \\
\frac{\partial \mathbf{K}_{T T}}{\partial \mathbf{x}} \cdot \mathbf{T}+\frac{\partial \mathbf{C}_{T T}}{\partial \mathbf{x}} \cdot \mathbf{T}+\frac{\partial \mathbf{L}_{T T}}{\partial \mathbf{x}} \cdot \mathbf{T}
\end{array}\right\}
\end{aligned}
$$

The derivative of the residual vector with respect to the design and state field in Eqs. (26) is achieved by assembling each term separately, e.g.:

$\frac{\partial \mathbf{M}_{P P}}{\partial \mathbf{x}} \cdot \dot{\mathbf{P}}=\sum_{i \in \mathbb{N}} \frac{\partial \mathbf{m}_{P P}^{e}}{\partial x^{e}} \cdot \dot{\mathbf{p}}^{\mathbf{e}}, \quad \ldots$

where ... denotes the remaining terms in Eq. (26).

The sensitivities in Eq. (24) are computed with the time stepping schemes in Eq. (17). The state fields and the adjoint fields are computed in opposite directions in time. As the state fields are computed for $j=\{1,2, \ldots N-1, N\}$, the adjoint fields are computed for $j=\{N, N-1, \ldots 2,1\}$. To compute the state fields and the adjoint fields are sometimes called the forward problem and the backward problem, respectively.

\subsubsection{Heat flux}

The objective of the design problem studied in this paper is to maximize the $x$-directional heat flux on the eastern boundary, $\Gamma^{E}$, during the discharge phase $d c h$, see Figs. $1 \mathrm{~b}$ and $1 \mathrm{c}$.

The inner objective function, $G=G(x, P, T)$, for the heat flux objective function is given by:

$G=\int_{\Gamma^{E}} Q_{x} \mathrm{~d} S$

where ${ }_{x}$ refer to the $x$-directional heat flux. Eq. (28) can be rewritten to:

$G=\sum_{k \in \mathbb{O}} \mathbf{Q}$

where $k$ is the $x$-directional heat flux for the $k$ 'th element on $\Gamma^{E}$ in the index set $\mathbb{O}$ and $\mathbf{Q}$ is the heat flux field. The objective function in Eq. (29) can be written in implementation form as:

$G=\mathbf{L}_{\mathbb{O}}^{T} \mathbf{Q}$
$\mathbf{L}_{\mathbb{O}}^{T}$ is a vector consisting of zeroes except for the positions $k \in \mathbb{O}$ which have the value $1 / l_{y, k}$, where $l_{y, k}$ is the height of the $k$ 'th element.

We need the gradients of the objective function with respect to the design field, $\partial G / \partial \mathbf{x}$ to compute the sensitivities in Eq. (25). These are given by:

$$
\frac{\partial G}{\partial \mathbf{x}}=\mathbf{L}_{\mathbb{O}}^{T} \frac{\partial \mathbf{Q}}{\partial \mathbf{x}}
$$

The inner objective function, $G$, with respect to the state variables, $\mathbf{S}$, is given by:

$\frac{\partial G}{\partial \mathbf{S}}=\left\{\begin{array}{l}\mathbf{L}_{\mathbb{O}}^{T} \frac{\partial \mathbf{Q}}{\partial \mathbf{P}} \\ \mathbf{L}_{\mathbb{O}}^{T} \frac{\partial \mathbf{Q}}{\partial \mathbf{T}}\end{array}\right\}$

The derivative of the heat flux with respect to the design and state field in Eqs. (31) and (32) are simply obtained with a finite element assembly:

$$
\frac{\partial \mathbf{Q}}{\partial \mathbf{x}}=\sum_{i \in \mathbb{N}} \frac{\partial \mathbf{q}^{e}}{\partial x} \quad \frac{\partial \mathbf{Q}}{\partial \mathbf{S}}=\sum_{i \in \mathbb{N}} \frac{\partial \mathbf{q}^{e}}{\partial \mathbf{s}}
$$

where $\mathbf{q}^{e}$ is the element heat flux, $x$ is the element design variable and $\mathbf{s}$ denotes the element state fields.

To solve the design problem in Eq. (18) for the inner objective function in Eq. (28), the terms in Eqs. (23), (25), (29), (30) and (31) are required.

Please notice that the terms presented in this section are dependent on the specific design problem. To derive the sensitivities for a design problem with a different objective functions requires actions in Eqs. (28), (30), (31) and (32).

\subsection{Filters and Projection Strategy}

The physical design variables, $\overline{\tilde{\rho}}_{i}$, are used in the finite element analysis and are obtained by the projection (Wang et al., 2011)

$\overline{\tilde{\rho}}_{i}=\frac{\tanh (\beta \eta)+\tanh \left(\beta\left(\tilde{\rho}_{i}-\eta\right)\right)}{\tanh (\beta \eta)+\tanh (\beta(1-\eta))}$

where $\eta$ is the projection filter threshold. The filtered design variables $\tilde{\rho}_{i}$ are obtained from the mathematical design variables, $\rho_{i}$, by the filter operation:

$\tilde{\rho}_{i}=\frac{\sum_{j \in \mathbb{N}_{i}} w\left(\mathbf{x}_{j}\right) v_{j} \rho_{j}}{\sum_{j \in \mathbb{N}_{i}} w\left(\mathbf{x}_{j}\right) v_{j}}$

where $v_{j}$ is the area of the $j$ 'th element, $\mathbb{N}_{i}$ is the index set of the design variables which are within the radius $R$ of design variable $i, w(\mathbf{x})$ is the filter weighting function and $x_{i}$ and $x_{j}$ are the spatial location of elements $i$ and $j$. The filter weighting function is given by: 
$w\left(\mathbf{x}_{j}\right)=\left\{\begin{array}{cc}R-|\mathbf{x}| & \forall|\mathbf{x}| \leq R \wedge \mathbf{x} \in \Omega_{D} \\ 0 & \text { otherwise }\end{array}\right.$

where $R$ is the filter radius, $|\mathbf{x}|=x_{i}-x_{j}$.

Finally, gradients with respect to design variables, $\rho_{i}$, require a transformation of the sensitivities by the chain rule:

$\frac{\partial L}{\partial \rho_{i}}=\sum_{j \in \Omega_{D}} \frac{\partial f}{\partial \overline{\tilde{\rho}}_{j}} \frac{\partial \overline{\tilde{\rho}}_{j}}{\partial \tilde{\rho}_{j}} \frac{\partial \tilde{\rho}_{j}}{\partial \rho_{i}}$

\section{Implementation}

To validate the finite element model and the sensitivities are critical parts of developing a topology optimization methodology. In this section we have validated the finite element model in Sec. 5.1, validated the sensitivities in Sec. 5.2, listed important implementation details in Sec. 5.3 and commented on the reproducibility of the design methodology in Sec. 10.

\subsection{Validation of the finite element model}

The finite element model laid out in Sec. 3 is validated by separately considering the convective and conductive heat transfer in two different and analytically solvable problems.

By extending the inlet and outlet over the entire side lengths of the storage matrix in Fig. 1a, the $y$-directional gradients of the pressure and temperature field can be neglected and the corresponding one-dimensional version of the design problem is achieved.

The validation study for the conductive and convective heat transfer takes basis in this problem and the study is carried out for the following academic model parameters: $\rho=2, c_{p}=0.5, k=1$ (conduction), $k=0$ (convection) and $L=2$.

\subsubsection{Conduction}

The time-dependent conductive heat transfer response in Eq. (1b) can be solved analytically by using the separation of variables approach for boundary value problems of partial differential equations (Strauss, 2007).

Assuming that the boundary and initial conditions of the conductive heat transfer validation study are $T(0, t)=T^{E}=1, T(L, t)=0$ and $T(x, 0)=0$, the temperature, $T=T(x, t)$, as function of the spatial coordinate, $x$, and the time, $t$, is given by the following expression:

$$
\begin{array}{r}
T(x, t)=\sum_{n=1}^{N}\left[\frac{2 c_{p} \rho T^{E} L^{2}}{k n^{3} \pi^{3}}\left(1+\frac{t k n^{2} \pi^{2}}{L^{2}}+\mathrm{e}^{-\frac{k n^{2} \pi^{2} t}{L^{2}}}\right)\right. \\
\left.\sin \left(\frac{n \pi x}{L}\right)\right]
\end{array}
$$

The exact solution to Eq. (38) is achieved for $N \rightarrow \infty$.

By comparing the analytic solution evaluated for $N=1000$ with the finite element model in Fig. 2, we point out that the modeling approaches almost provide perfectly equivalent result. With reference to the excellent fit between the modeling approaches, we confidently conclude that the convective heat transfer problem is adequately resolved with the finite element model proposed in Sec. 3.

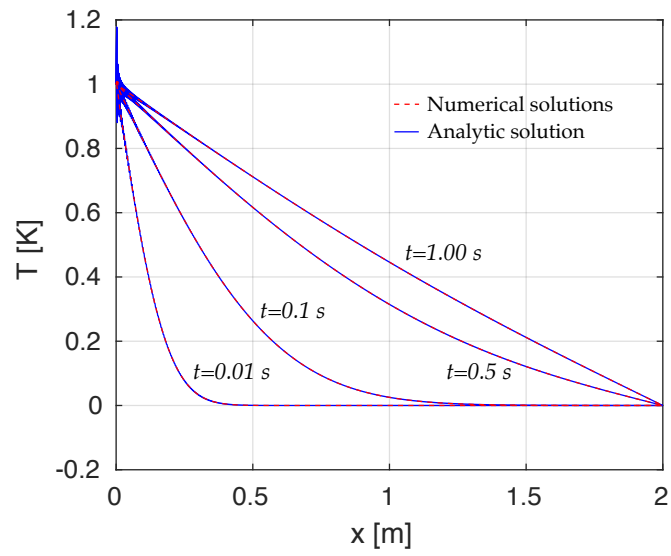

Fig. 2: A comparison between the analytical and numerical models for conductive heat transfer. The temperature, $T$, is plotted as function of the spatial coordinate $x$ for different times $t$. Due to the excellent fits between the models, we conclude that the heat transfer due to conduction is adequately resolved in the numerical model.

\subsubsection{Convection}

The time-dependent convective heat transfer response in Eq. (1b) can be solved analytically by assuming that the transition in temperature between the hot heat transfer fluid and the initial temperature of the storage matrix remains perfectly abrupt (McTigue, 2016). This transition is from now on denoted the transition zone.

By setting the thermal conductivity to zero, it is possible to determine the position of the thermal front, $s$, as function of time, $t$, by:

$s(t)=\frac{\kappa}{\mu} \Delta p t$ 
where $\Delta p$ denotes the pressure difference between $\Gamma^{E}$ and $\Gamma^{W}$

By using Eq. (39) and the boundary and initial conditions, $T(0, t)=1$ and $T(x, 0)=0$, it is now possible to determine the temperature, $T$, as function of the spatial coordinate, $x$, for different times, $t$. This analytic relationship has been compared with the numerical model in Fig. 3. With reference to the figure, it is seen that the numerical model provides solutions with considerably larger extensions of the transition zones than the analytic model. This issue is caused by a compromise between three conflicting modeling technicalities.

The stabilization term in Eq. (8e) is basically an artificial conduction-contribution, which is added to the thermal problem in Eq. (1b) in order to damp nonphysical oscillations in the temperature field for convection dominated problems. The non-physical temperature oscillations are successfully removed with this approach but on behalf of an increased extent of the transition zone.

Implicit time-stepping schemes are generally prone to numerical damping (Cook et al., 2007). Numerical damping in convective heat transport problems is expressed as an artificial conduction-contribution and an increased extent of the transition zone. Explicit schemes are generally better suited for capturing large gradients in the state fields, such as a narrow transition zone, however, these schemes suffer from non-physical oscillations in the temperature field. To avoid these oscillations, considerably more stabilization is required which result in a transition zone somewhat equivalent to the implicit time stepping schemes.

Implicit time stepping schemes are unconditionally stable and computationally cheep memory-wise for large time steps, however large time steps reduce non-physical oscillations in the temperature field but on behalf of an increased amount of artificial conduction.

The numerical examples in this paper are based on a comprehensive study and parameter calibration which take the above considerations into account and solves the state problems with an acceptable accuracy.

The finite element model has also been successfully verified for convective and conductive heat transfer with the commercial finite element software, COMSOL. With reference to the validation studies in Sec. 5.1.1 and 5.1.2 and the successful COMSOL validation study, we conclude that the finite element model is reliable.

\subsection{Validation of sensitivities}

To validate the sensitivities is a critical part of the development of a topology optimization methodology and

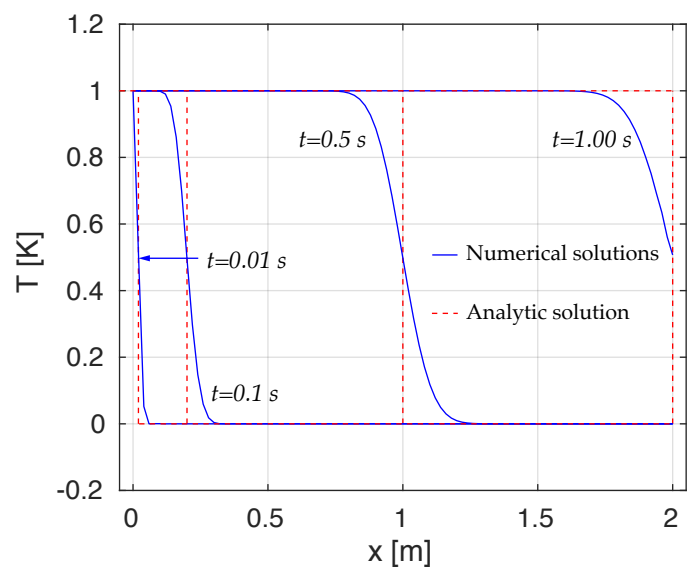

Fig. 3: A comparison between the analytic and numerical models for convective heat transfer in porous media. The figure shows the temperature, $T$, plotted as function of the spatial coordinate, $x$, for different times, $t$. Due to the acceptable fits between the modeling approaches, we conclude that the convective heat transfer is adequately resolved by the numerical model.

framework. A validation study of the sensitivities is performed by comparing the analytic adjoint sensitivities with a finite difference approximation to the sensitivities. The error in element $i$ between the analytic, $\mathrm{d} L / \mathrm{d} x_{i}$, and approximate sensitivities, $\mathrm{d} L_{a p p} / \mathrm{d} x_{i}$, is given by:

$\epsilon_{i}=\left|\frac{\mathrm{d} L / \mathrm{d} x_{i}-\mathrm{d} L_{a p p} / \mathrm{d} x_{i}}{\mathrm{~d} L / \mathrm{d} x_{i}}\right|$

where $\|\square\|$ denotes the absolute value of $\square$. The approximation to the sensitivities for element $i$ is computed with the following finite difference scheme:

$\frac{\mathrm{d} L_{a p p, i}}{\mathrm{~d} x_{i}}=\frac{f\left(x_{i}\right)-f\left(x_{i}+\Delta x\right)}{\|\Delta x\|}$

where $\Delta x$ denotes a small perturbation in the design field. To achieve the approximate sensitivity field it is required to evaluate Eq. (41) for all elements which is computational expensive compared to computing the analytic adjoint sensitivities in Eq. (24).

The error between the approximate and analytic sensitivities for a sequence of different perturbations in the design field, $\Delta x=\left\{10^{-1}, 10^{-2}, \ldots, 10^{-7}, 10^{-8}\right\}$ have been plotted in Fig. 4. The validation study is conducted on all elements (for a coarsely discretized problem), a design field with randomly chosen design variables and the design problem sketched in Fig. 1. As the validation study is conducted for all elements, each curve on the figure represents the convergence of error for one element. To validate all elements is important because an error in the sensitivity analysis may not necessarily be expressed in all elements. 
As we observe excellent convergence of the errors, we confidently conclude that the sensitivity analysis is correctly derived and implemented.

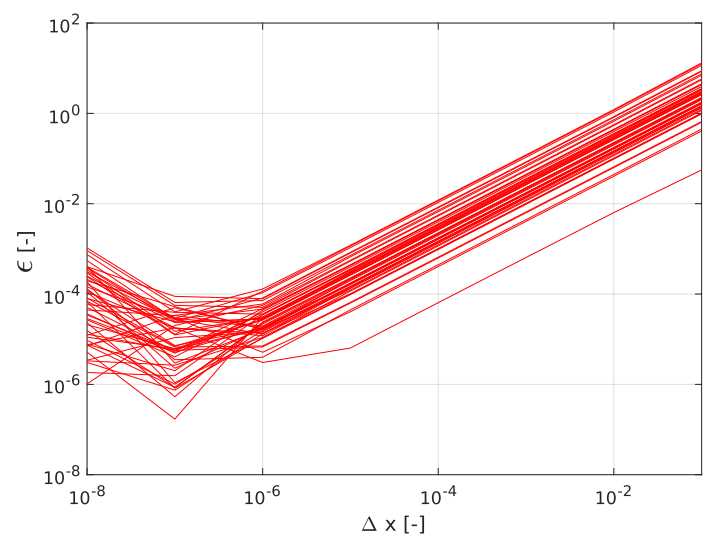

Fig. 4: The error, $\epsilon$, between the analytic and approximated sensitivities plotted as function of the magnitude of the perturbation in the design field, $\Delta x$. The analytic and approximated sensitivities are computed with the adjoint sensitivity analysis in Sec. 4 and a backward finite difference approximation in Eq. (41), respectively. Despite the time-dependence, weakly coupled physics and a randomly fluctuating design field, the errors have a linear convergence rate (in a logarithmic scale) and a small magnitude for all elements which indicate that the sensitivity analysis is correctly derived and implemented.

\subsection{Design problem details}

The material parameters of the sand and rocks/gravel in Fig. 1 and Tab. 2 are the temperature independent version of the temperature dependent material parameters numerically and experimentally validated in the work of Bruch et al. (2014). The material parameters are suited for the design problem solved in the present paper for the following reasons: (1) the assumption about incompressibility in Eq. (1a) is adequate, as the thermal heat transfer fluid is in the liquid phase (oil) where the bulk modulus is large. (2) As the difference between the extreme limits of the temperature is approximately 100 K (Bruch et al., 2014), the importance of taking temperature dependent material parameters into account may be less critical. Furthermore, we argue that the assumption about temperature independent material parameters is acceptable, as the difference between the extreme limits of the material parameters in the relevant temperature range are: $14 \%, 4 \%, 55 \%$ and $9 \%$ for $c_{p}, k$,
Table 2: A list of model parameters used to solve the design problem.

\begin{tabular}{|c|c|c|}
\hline \multicolumn{3}{|l|}{$\begin{array}{l}\text { Material parameters } \\
\text { (rocks/gravel) }\end{array}$} \\
\hline $\begin{array}{l}\text { Heat capacity } \\
\text { Conductivity } \\
\text { Viscosity } \\
\text { Density } \\
\text { Permeability } \\
\end{array}$ & $\begin{array}{r}c_{p}=2.267 \\
k_{s}=0.117 \\
\mu_{s}=6.10 \cdot 10^{-4} \\
\rho_{s}=846.72 \\
\kappa_{s}=10^{-7} \\
\end{array}$ & $\begin{array}{r}{[\mathrm{J} / \mathrm{kg} \cdot \mathrm{K}]} \\
{[\mathrm{W} / \mathrm{K} \cdot \mathrm{m}]} \\
{[\mathrm{Pa} \cdot \mathrm{s}]} \\
{\left[\mathrm{kg} / \mathrm{m}^{3}\right]} \\
{\left[\mathrm{m}^{2}\right]}\end{array}$ \\
\hline \multicolumn{3}{|l|}{$\begin{array}{l}\text { Material parameters } \\
\text { (sand) }\end{array}$} \\
\hline $\begin{array}{l}\text { Heat capacity } \\
\text { Conductivity } \\
\text { Viscosity } \\
\text { Density } \\
\text { Permeability }\end{array}$ & $\begin{array}{r}c_{p}=900 \\
k_{w}=0.1 \\
\mu_{w}=6.10 \cdot 10^{-4} \\
\rho_{w}=2500 \\
\kappa_{w}=10^{-11}\end{array}$ & $\begin{array}{r}{[\mathrm{J} / \mathrm{kg} \cdot \mathrm{K}]} \\
{[\mathrm{W} / \mathrm{K} \cdot \mathrm{m}]} \\
{[\mathrm{Pa} \cdot \mathrm{s}]} \\
{\left[\mathrm{kg} / \mathrm{m}^{3}\right]} \\
{\left[\mathrm{m}^{2}\right]}\end{array}$ \\
\hline \multicolumn{3}{|l|}{$\begin{array}{l}\text { Dimensions } \\
\text { of design problem }\end{array}$} \\
\hline $\begin{array}{l}\text { Length of storage matrix, } x \\
\text { Height of storage matrix, } y \\
\text { Number of elements, } x \\
\text { Number of elements, } y \\
\text { Number of time steps } \\
\text { Time step length }\end{array}$ & $\begin{array}{r}L_{x}=2 \\
L_{y}=1 \\
200 \\
100 \\
144 \\
\Delta t=25\end{array}$ & $\begin{array}{r}{[\mathrm{m}]} \\
{[\mathrm{m}]} \\
{[-]} \\
{[-]} \\
{[-]} \\
{[\mathrm{s}]} \\
\end{array}$ \\
\hline \multicolumn{3}{|l|}{$\begin{array}{l}\text { Parameters for the } \\
\text { charging phase }\end{array}$} \\
\hline $\begin{array}{l}\text { Start of charge phase } \\
\text { End of charge phase }\end{array}$ & $\begin{array}{r}T_{c h}^{E}=400 \\
P_{c h}^{W}=\{3500 \\
6000,9000\} \\
P_{c h}^{W}=0 \\
T_{0}=0 \\
T_{S}=1800\end{array}$ & $\begin{array}{r}{[\mathrm{K}]} \\
\\
{[\mathrm{Pa}]} \\
{[\mathrm{Pa}]} \\
{[\mathrm{s}]} \\
{[\mathrm{s}]}\end{array}$ \\
\hline \multicolumn{3}{|l|}{$\begin{array}{l}\text { Parameters for the } \\
\text { discharging phase }\end{array}$} \\
\hline $\begin{array}{l}\text { Start of discharge phase } \\
\text { End of discharge phase }\end{array}$ & $\begin{array}{r}T_{d c h}^{W}=300 \\
P_{d c h}^{W}=\{3500 \\
6000,9000\} \\
P_{d c h}^{E}=0 \\
T_{S}=1800 \\
T_{E}=3600\end{array}$ & $\begin{array}{r}{[\mathrm{K}]} \\
{[\mathrm{Pa}]} \\
{[\mathrm{Pa}]} \\
{[\mathrm{s}]} \\
{[\mathrm{s}]}\end{array}$ \\
\hline
\end{tabular}

$\mu$ and $\rho$. Please notice that the color map used to present design solutions is chosen such that blue corresponds to gravel/rocks and yellow corresponds to sand.

The Heaviside projection parameter, $\beta$, in Eq. (34) is updated every 100th design iteration after the scheme: $\beta=\{4,8,16,32,64,128\}$. The design process is stopped when $\beta=128$ and the design process is converged, i.e. when the maximum difference between the design variables in iteration $k$ and $k-1$ is less than $0.1 \%$.

The interpolation function parameters in Eq. (12) are $p_{k}=1, p_{\kappa}=10^{-4}, p_{c_{p}}=1$ and $p_{\rho}=1$. These parameters have large influence on the well-posedness of the 
design problem. The issue is discussed for fluid-structureinteraction problems in Lundgaard et al. (2018a).

The optimization problems are solved using the method of moving asymptotes (Svanberg, 1987) with the standard settings and an outer move limit of 0.25 . The finite element equations and sensitivities are derived in the mathematical software package, Maple, and implemented in mathematical scripting language, Matlab.

For clarity purposes, we have listed the material and model parameters of the design problem in Tab. 2.

\section{Numerical examples}

To demonstrate the capability of the methodology presented, we solve a design problem for an industrial and physically realistic TES system. The design problem can be seen in Fig. 1 and is inspired by the work of Bruch et al. (2014).

The design solutions solved for the heat flux objective in Eq. (11) and different magnitudes of the pressure driving the flow during the charging and discharging phases, $\Delta P$, have been plotted in Fig. 5 .

With reference to the figure, the design solutions can generally be characterized by three attributes: $(\mathrm{A})$ the ratio between the of sand and the gravel/rocks, (B) the complexity of the heat flow path and $(\mathrm{C})$ the surface area of the transition between the sand and gravel/rocks material phases.

As the pressure difference is increased; the velocity of the fluid, the convective heat transfer, the amount of sand and the complexity of the heat flow paths are increased. The topology of the sand phase is used to control the time it takes for the heat to travel between the eastern and western boundaries. The complexity of the topology of the sand phase is a compromise between two conflicting parameters: Too simple topologies do not take advantage of the large heat capacity of the sand phase. Too complex topologies result in a poorly filled storage matrix, due to the too long and complex flow path. The topology of the design solutions in Fig. 5 are constructed such that a good compromise between these effects are found, which results in a well-performing design solution.

The ratio between the convective and the conductive heat transfer are controlling the feature sizes of the material phases. The feature size of the sand material phases are large for small pressure differences, as the ratio between the conductive and convective heat transfer is relatively small for small pressure differences. The large surface area of the design solutions in Fig. $5 \mathrm{c}$ is used to increase the effectiveness of the conductive heat transfer in the convective dominated flow.
To make probable that the design solutions in Fig. 5 indeed have superior performance for the model parameters they were optimized for, we have carried out a so-called cross-check. Cross-checks are very important in many aspects of optimization, as they en-light how much significance we can attribute to the features of the design solutions.

The cross-check table in Tab. 3 contains the evaluations of the heat flux for all combinations of the design solutions in Fig. 5 and the magnitude of the pressure driving the fluid flow in the charging and discharging phases, $\Delta P$.

The objective functions are evaluated for projected binary $(0 / 1)$ designs using $x=0.5$ as threshold value. This sharp thresholding is carried out to ensure that the improved performances of the optimized designs are not governed by nonphysical intermediate design variables which can occur due to the large differences between the material parameters of the material phases in Tab. 2 .

As the largest magnitudes of the heat fluxes are in the diagonal of the cross check table, we confidently conclude that $\Delta P$ is an important model parameter and that the design methodology captures the effect of the parameter in the design solutions.

Table 3: Cross-check between the heat flux objective function in Eq. (11) for the design solutions in Fig. 5 and the pressure driving the fluid flow, $\Delta P$. As the best performing designs are achieved for the model parameters at which they were optimized, we confidently conclude that $\Delta P$ is a critical model parameter and that the significance of the parameter is captured adequately in the design solutions.

\begin{tabular}{|c|c|c|c|}
\hline \multirow{2}{*}{$\begin{array}{l}\text { Design } \\
\text { optimized for }\end{array}$} & \multicolumn{3}{|c|}{ Evaluated for } \\
\hline & $\Delta P=3500$ & $\Delta P=6000$ & $\Delta P=9000$ \\
\hline$\Delta P=3500$ & $17.00 \cdot 10^{7}$ & $17.99 \cdot 10^{7}$ & $18.04 \cdot 10^{7}$ \\
\hline$\Delta P=6000$ & $16.42 \cdot 10^{7}$ & $21.35 \cdot 10^{7}$ & $21.96 \cdot 10^{7}$ \\
\hline$\Delta P=9000$ & $12.47 \cdot 10^{7}$ & $19.98 \cdot 10^{7}$ & $23.80 \cdot 10^{7}$ \\
\hline
\end{tabular}

To demonstrate the features of the design solutions, we have plotted the temperature fields for the design solution in Fig. 5c during the charging phase in Fig. 6 and the discharging phase in Fig. 7. The specific times at which the temperature fields are plotted is equally distributed in the entire time series, i.e. $t \in\left[0 ; T_{E}\right]$.

With reference to the figures, we notice the following features of the design solution and the corresponding temperature response: In accordance to the material parameter listed in Tab. 2, the heat transfer is primarily governed by convection in the gravel/rocks material 


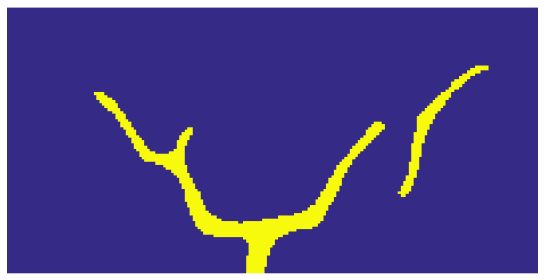

(a) $\Delta P=3500$

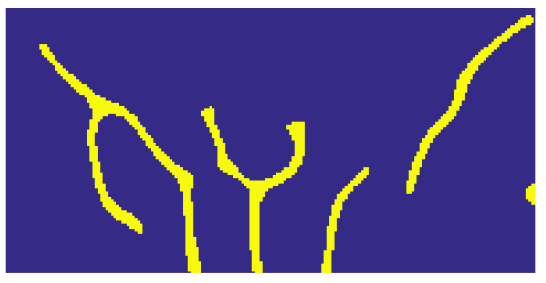

(b) $\Delta P=6000$

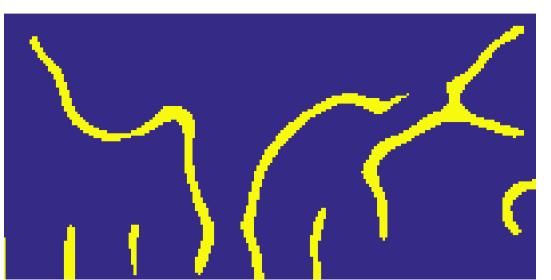

(c) $\Delta P=9000$

Fig. 5: Design solutions solved for different magnitudes the pressure driving the flow during the charging and discharging phases, $\Delta P$. The design solutions depend on $\Delta P$ which indicate that this is an important model parameter for the material layout of sand and gravel/rocks material phases in the storage matrix of TES systems.

phase and by conduction in the sand phase. As the ratio between the convective and conductive heat transfer is large, the surface area of the transition between the material phases is large. This feature is used to effectively absorb and extract the thermal energy in the sand phases during the re-generation cycle.

As the heat flow paths in the charging and discharging phases are different, it is necessary to model the full cycle to fully capture the features of the underlying physics.

To further validate the design solutions, we have compared the objective functions for design solution in Fig. 5c and a benchmark design consisting exclusively of the gravel/rocks material phase. As the design consisting exclusively of gravel/rocks perform better than the design consisting exclusively of sand for the model parameters in this study, we have decided to use this design as the benchmark.

The temperature, $T$, and the $x$-directional heat flux $Q_{x}$ as function of the time, $t$, have been plotted in Fig. 8 for the benchmark design and the design solution in Fig. 5c.

By computing the objective function for the benchmark and the design solution in Fig. 5c, i.e. integrating under the curves in Fig. 8 for time interval $\left.t \in] T_{S} ; T_{E}\right]$, the performance of the topology optimized design solution is $\approx 46 \%$ higher than the benchmark design. The better performance of the topology optimized design solutions are conditioned by the more complex flow path and the thermal properties of the sand phase which can store more energy per unit volume compared to the gravel/rocks material phase.

To demonstrate the behavior of the design algorithm, we have in Fig. 9 plotted the normalized heat flux objective, $f_{Q}$, as function of design iteration number, $k$ for the design solution in Fig. 5c. A sequence of snapshots of the design evolution has been plotted in Fig. 10. We notice that the convergence of the design problem is smooth and stable despite the time dependent and weakly coupled physics. The discontinuities in the convergence plot are caused by the updates of the threshold projection parameter, $\beta$, in Eq. (34). 


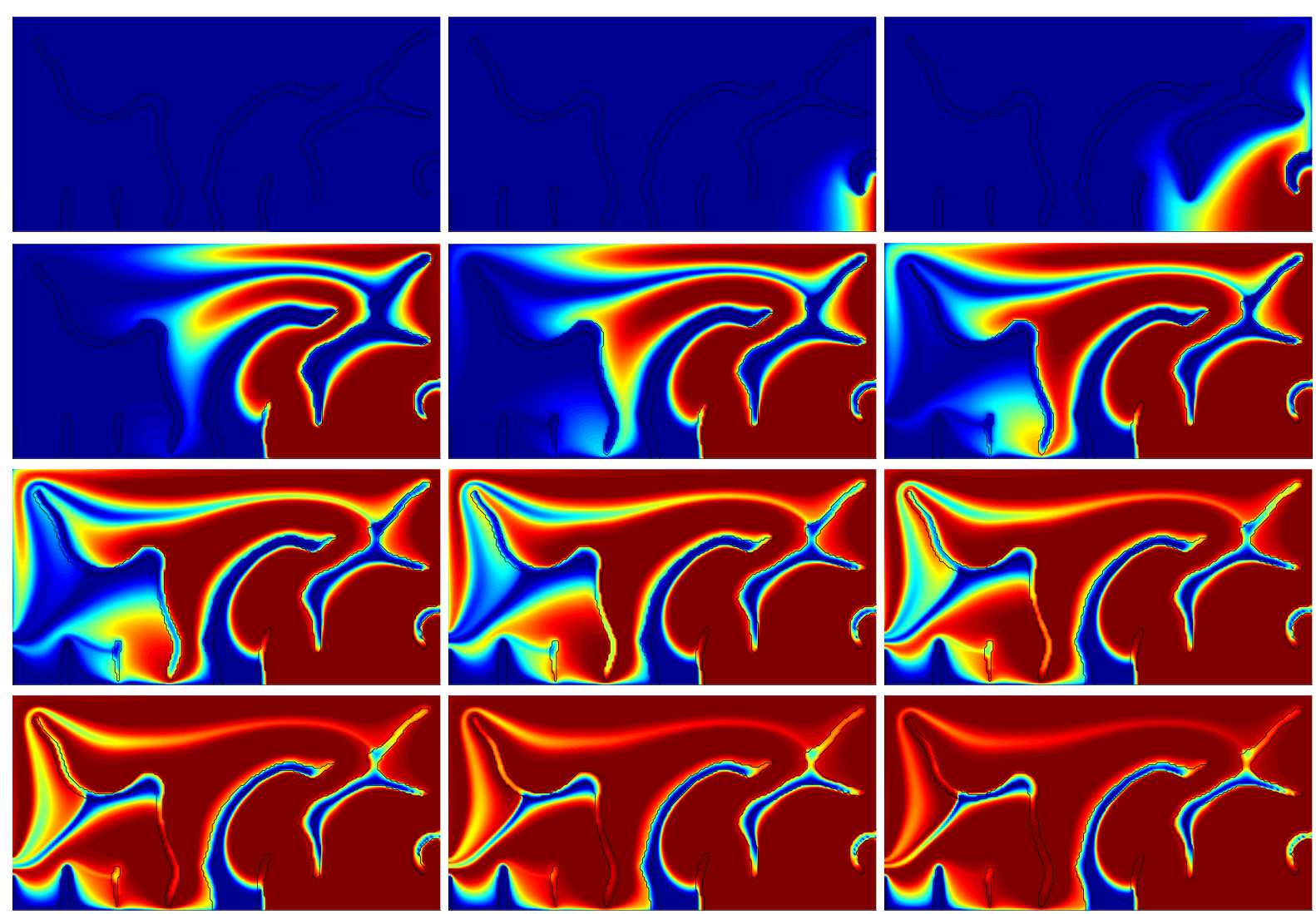

Fig. 6: The temperature state fields for the design solution in Fig. 5c plotted for different time steps during the charging phase.
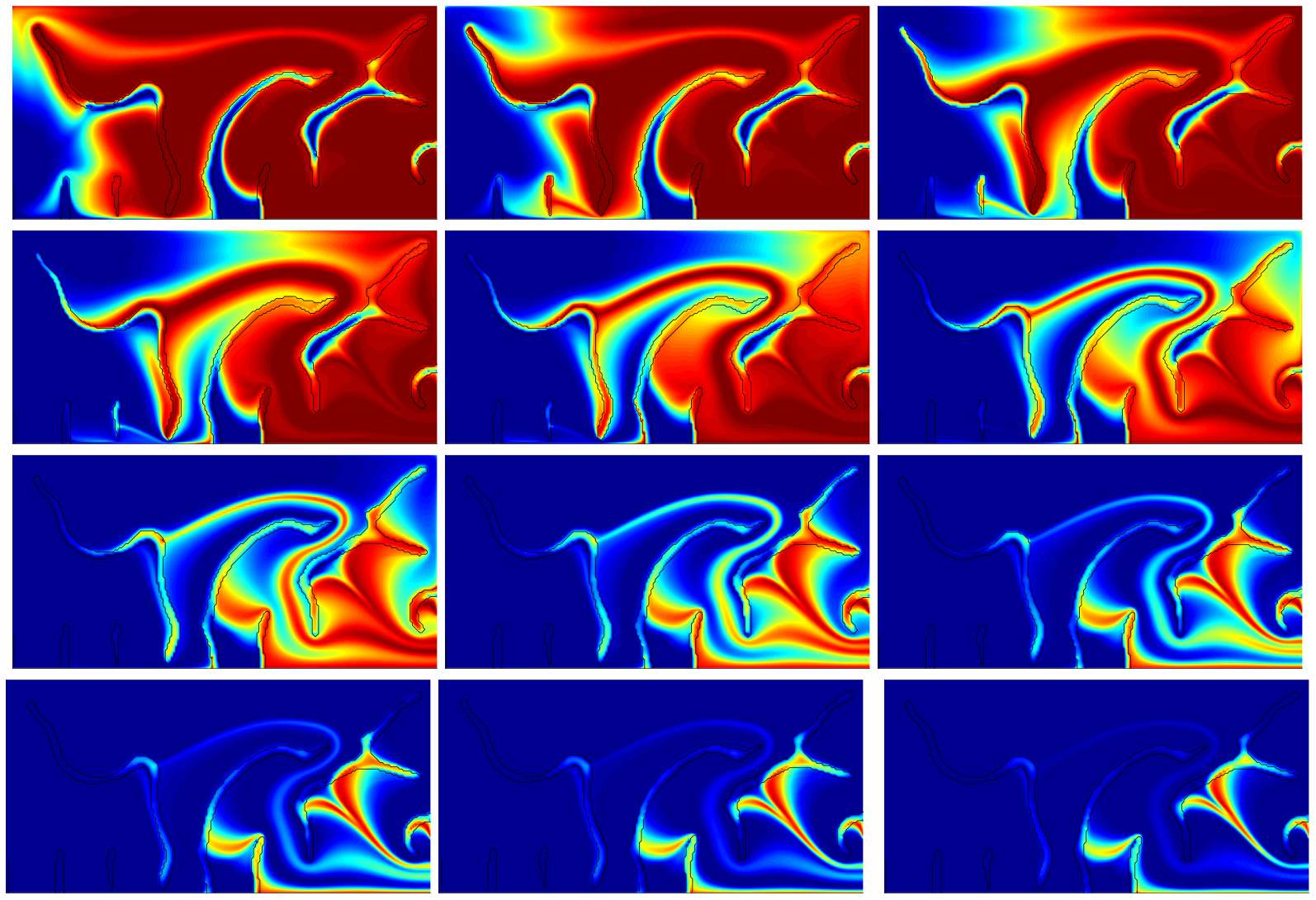

Fig. 7: The temperature state fields for the design solution in Fig. 5c plotted for different time steps during the discharging phase. 


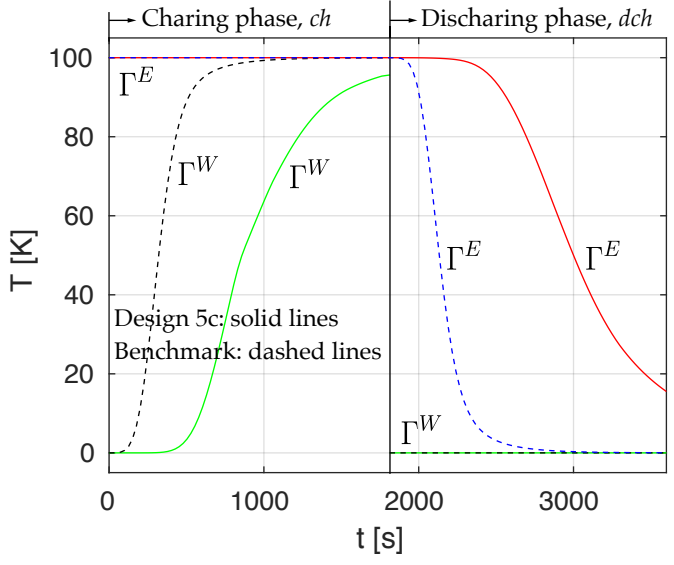

(a) Temperature, $T$

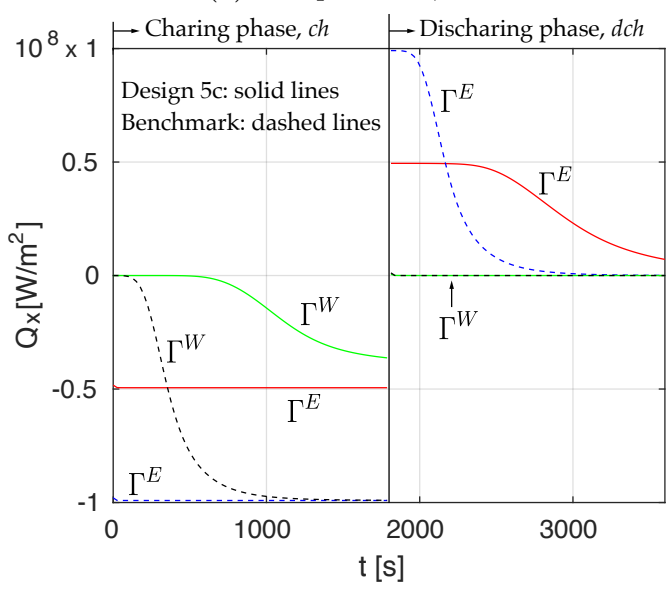

(b) $x$-directional heat flux, $Q_{x}$

Fig. 8: The temperature, $T$, and the $x$-directional heat flux, $Q_{x}$, as function of time, $t$, evaluated at $\Gamma^{E}$ and $\Gamma^{W}$ for benchmark design solutions and the design solution in Fig. 5c. With respect to the objective function in Eq. (11), the performance of the topology optimized design solution is $\approx 46 \%$ higher than the benchmark design.

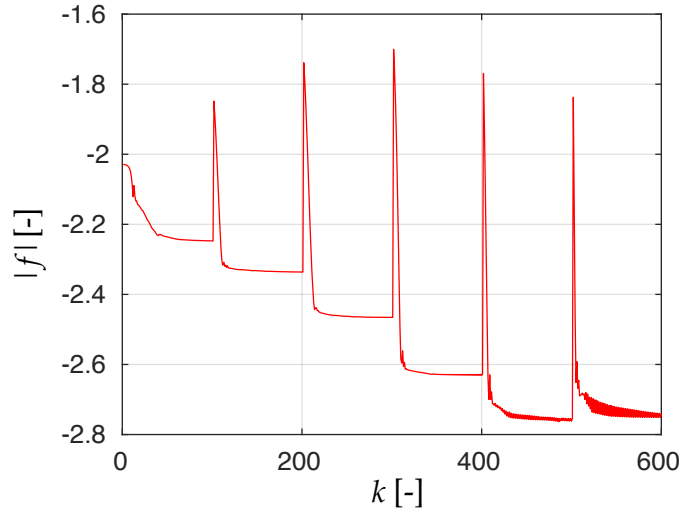

Fig. 9: The normalized objective, $f$, plotted as function of the design iteration number, $k$, for the design solution in Fig. 5c. The discontinuities in the convergence plot are caused by the updates of the threshold projection parameter, $\beta$, in Eq. (34). 


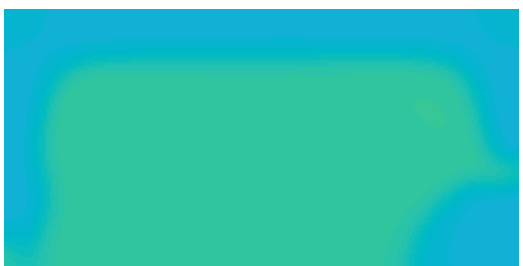

(a) $k=1$

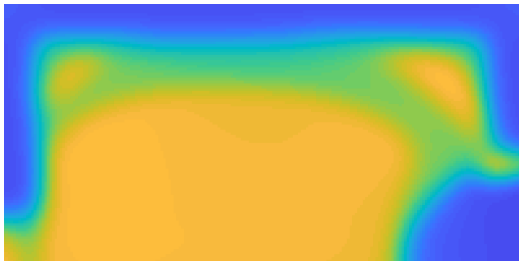

(b) $k=50$

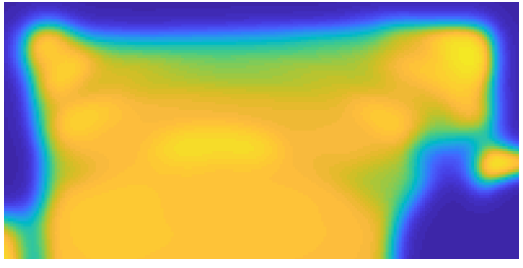

(c) $k=100$

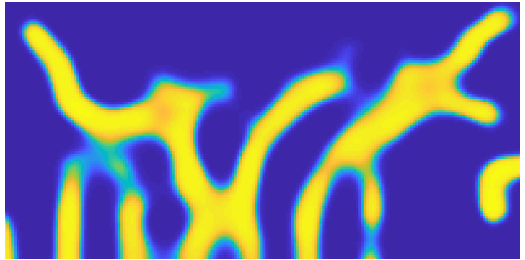

(d) $k=200$

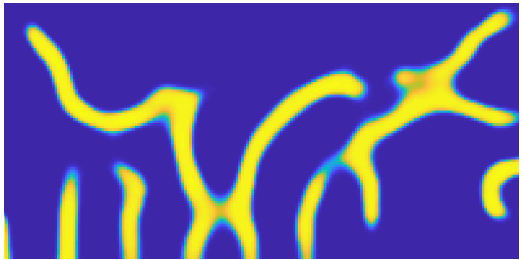

(e) $k=300$

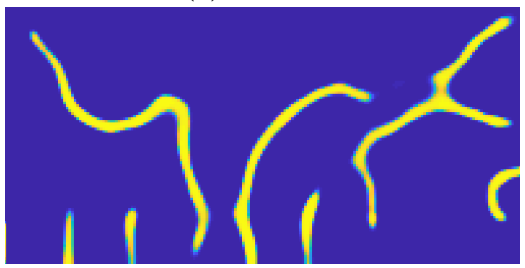

(f) $k=450$

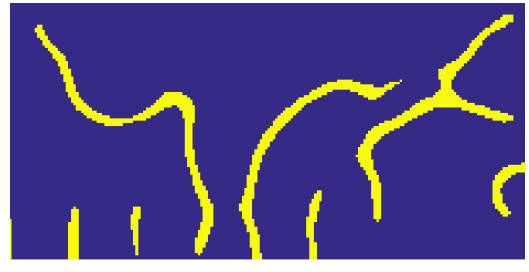

(g) $k=600$

Fig. 10: Snapshots of the design evolution which result in the design solution in Fig. 5c. 


\section{Discussion}

All results presented in the present paper are supported by validation studies, benchmark examples and crosschecks, for which reason we confidently conclude that the topology optimization approach presented in the present study is a promising methodology for solving the design problem in Eq. (18). In the following sections we have discussed some important topics related to the methodology.

\subsection{The choice of objective function}

During the development of the methodology, we have investigated various objective functions. We found that the objective function in Eq. (30) is better suited for optimizing the performance of TES systems than (A) the average temperature on $\Gamma^{E}$ during the discharge phase or (B) the ratio between the amount of thermal energy entering and exiting $\Gamma^{E}$ during the charging and discharging phases, respectively. As there are no inherent feature in these alternative objective functions which ensure that a large amount of thermal energy is extracted from the storage matrix during the discharge phase, we concluded that these objective functions are unsuited for the design problems investigated in the present paper.

\subsection{Design problems are dependent on the cycles}

As the heat flow path in the charging phase is different from the flow path in the discharging phase, it is necessary to model the full re-generation cycle. To take multiple charging and discharging phases into consideration may be an important future study, as industrial applications of re-generators undergo large numbers of charging and discharging cycles in their operation.

\subsection{Temperature independent materials}

The design problems were solved for temperature independent material parameters, which indeed is an inadequate assumption for some physical materials. We decided to limit the design problems to temperature independent materials to reduce the complexity of the design solutions and the computational costs. To take temperature dependent material parameters into consideration can straight forwardly be achieved by consulting e.g. Lundgaard and Sigmund (2018).

\subsection{Phase change materials}

TES systems can be divided into two categories: sensible and latent. Sensible heat systems store energy in a medium by increasing the temperature. Latent heat systems store energy by changing the phase of the material. In the present study we have focused on sensible systems, however the methodology can straight forwardly be extended to take phase changing materials into consideration, see e.g. the work of Lundgaard et al. (2018b), Pizzolato et al. (2017). Phase change materials can be taken into consideration by making the material parameters in Eq. (7) temperature-dependent and add the corresponding temperature variance to Eq. (15).

\subsection{Density variations}

The present study takes basis in a related set of material parameters found in the work of Bruch et al. (2014). Bruch et al. used an oil with low viscosity as the heat transfer fluid to charge the storage matrix with thermal energy. As the compressiblity for oil is low, we argue that it is reasonable to assume that the variance of the density with respect to time in Eq. (1a) is neglectable. However, the framework can easily be extended to take compressible fluid and natural convection into consideration if the term $\partial \rho / \partial t=0$ is added to Eq. (1a). As Eq. (1) in such case is strongly coupled, the computational cost would also increase considerably.

\section{Conclusion}

We have presented a generally applicable density-based topology optimization methodology which can be used to optimize the performance of a thermal energy storage system. The performance of the systems are optimized by spatially distributing two different materials with different thermal properties in a two dimensional design space. With basis in a potential fluid model coupled to thermal heat transfer, the methodology is derived and implemented and exhaustive implementation details are provided.

The most important findings of the study have been listed in the following:

1. With reference to the validation studies in Secs. 5.1 and 5.2 , we conclude that we can rely on the underlying mathematical model of the framework.

2. We conclude that the framework is suited for solving the design problem in Eq. (18), as the design solutions are validated by cross-check studies and benchmark designs and pass all tests. 
3. The design solutions obtained by the topology optimization methodology outperform the benchmark design solutions by $46 \%$. We therefore confidently conclude that the methodology is excellently suited for optimizing the storage matrix of thermal energy storage systems.

4. The study reveals new insight in physical and topological effects of thermal energy storage systems, as it is shown that the design solutions depend on the boundary conditions, the material parameters and the objective functions. To obtain high performing thermal energy storage systems, it is therefore critical to take these parameters into consideration.

The methodology can easily be extended to incorporate temperature dependent materials (phase change materials), multiple materials phases, other objective functions, boundary conditions and density variations (natural convection). These studies may be conducted in the future, but for now this paper serves as a demonstration that topology optimization is a suitable methodology for optimizing thermal energy storage systems.

\section{Acknowledgements}

The authors acknowledge the financial support received from the TopTen project sponsored by the Danish Council for Independent Research (DFF-4005-00320).

\section{Replication of results}

As comprehensive implementation details are provided, we are confident than the design methodology is reproducible, for which reason we have decided not the publish the code. If the information provided in the paper isn't sufficient, scientist or interested parties are welcome to contact the authors for further explanations.

\section{References}

Francis Agyenim, Neil Hewitt, Philip Eames, and Mervyn Smyth. A review of materials, heat transfer and phase change problem formulation for latent heat thermal energy storage systems (lhtess). Renewable and sustainable energy reviews, 14(2):615-628, 2010.

Joe Alexandersen, Niels Aage, Casper Schousboe Andreasen, and Ole Sigmund. Topology optimisation for natural convection problems. Int. J. Numer. Meth. Fluids, pages 699-721, 2014. doi: 10.1002/fld.

Martin Bendsøe and Ole Sigmund. Topology Optimization - Theory, Methods and Applications. Springer, 2003.
Martin Philip Bendsøe and Noboru Kikuchi. Generating optimal topologies in structural design using a homogenization method. Computer methods in applied mechanics and engineering, 71(2):197-224, 1988.

Thomas Borrvall and Joakim Petersson. Topology optimization of fluids in Stokes flow. International Journal for Numerical Methods in Fluids, 41(1):77-107, 2003.

A Bruch, JF Fourmigué, and R Couturier. Experimental and numerical investigation of a pilot-scale thermal oil packed bed thermal storage system for csp power plant. Solar Energy, 105:116-125, 2014.

Haisheng Chen, Thang Ngoc Cong, Wei Yang, Chunqing Tan, Yongliang Li, and Yulong Ding. Progress in electrical energy storage system: A critical review. Progress in natural science, 19(3):291-312, 2009.

Robert D. Cook, David S. Malkus, Michael E. Plesha, and Robert J. Witt. Concepts and applications of finite element analysis. John Wiley \& Sons, 4th edition, 2007.

Jonas Dahl, Jakob S. Jensen, and Ole Sigmund. Topology optimization for transient wave propagation problems in one dimension : DDesign of filters and pulse modulators. Structural and Multidisciplinary Optimization, 36(6):585-595, nov 2008. ISSN 1615147X. doi: 10.1007/s00158-007-0192-5.

Joshua D Deaton and Ramana V Grandhi. A survey of structural and multidisciplinary continuum topology optimization: post 2000. Structural and Multidisciplinary Optimization, 49(1):1-38, 2014.

Yongbo Deng, Zhenyu Liu, Ping Zhang, Yongshun Liu, and Yihui Wu. Topology optimization of unsteady incompressible Navier-Stokes flows. Journal of Computational Physics, 230(17):6688-6708, 2011.

Antoni Gil, Marc Medrano, Ingrid Martorell, Ana Lázaro, Pablo Dolado, Belén Zalba, and Luisa F Cabeza. State of the art on high temperature thermal energy storage for power generation. part 1concepts, materials and modellization. Renewable and Sustainable Energy Reviews, 14(1):31-55, 2010.

Jakob S Jensen, Praveen B Nakshatrala, and Daniel A Tortorelli. On the consistency of adjoint sensitivity analysis for structural optimization of linear dynamic problems. Structural and Multidisciplinary Optimization, 49(5):831-837, 2014.

David Kearney, Ulf Herrmann, Paul Nava, B Kelly, Rod Mahoney, J Pacheco, Robert Cable, N Potrovitza, Daniel Blake, and Henry Price. Assessment of a molten salt heat transfer fluid in a parabolic trough solar field. Journal of solar energy engineering, 125 (2):170-176, 2003.

Robert B Laughlin. Pumped thermal grid storage with heat exchange. Journal of Renewable and Sustainable Energy, 9(4):044103, 2017. 
Ying Li, Kazuhiro Saitou, and Noboru Kikuchi. Topology optimization of thermally actuated compliant mechanisms considering time-transient effect. Finite elements in analysis and design, 40(11):1317-1331, 2004.

Henrik Lund. Renewable energy strategies for sustainable development. Energy, 32(6):912-919, 2007.

Chrisitan Lundgaard, Joe Alexandersen, Mingdong Zhou, Casper Schoesboe, and Ole Sigmund. Topology optimization for fluid-structure-interaction problems. Structural and Multidisciplinary Optimization, 2018a. doi: 0.1007/s00158-018-1940-4.

Christian Lundgaard and Ole Sigmund. A density-based topology optimization methodology for thermoelectric energy conversion problems. Structural and Multidisciplinary Optimization, 57(4):1427-1442, 2018.

Christian Lundgaard and Ole Sigmund. Design of segmented off-diagonal thermoelectric generators using topology optimization. Applied Energy, 236:950-960, 2019.

Christian Lundgaard, Joe Alexandersen, Mingdong Zhou, Casper Schousboe Andreasen, and Ole Sigmund. Revisiting density-based topology optimization for fluid-structure-interaction problems. Structural and Multidisciplinary Optimization, pages 1-27, 2018b.

Joshua McTigue. Analysis and optimisation of thermal energy storage. PhD thesis, Department of Engineering, University of Cambridge, 2016.

RM Muthusivagami, R Velraj, and R Sethumadhavan. Solar cookers with and without thermal storagea review. Renewable and Sustainable Energy Reviews, 14 (2):691-701, 2010.

Sebastian Nørgaard, Ole Sigmund, and Boyan Lazarov. Topology optimization of unsteady flow problems using the lattice Boltzmann method. Journal of Computational Physics, 307:291-307, 2016.

Amaya V Novo, Joseba R Bayon, Daniel Castro-Fresno, and Jorge Rodriguez-Hernandez. Review of seasonal heat storage in large basins: Water tanks and gravelwater pits. Applied Energy, 87(2):390-397, 2010.

Fridolin Okkels and Henrik Bruus. Scaling behavior of optimally structured catalytic microfluidic reactors. Phys. Rev. E, 75:016301, 2007.

Claus BW Pedersen. Crashworthiness design of transient frame structures using topology optimization. Computer methods in applied mechanics and engineering, 193(6-8):653-678, 2004.

Alberto Pizzolato, Ashesh Sharma, Kurt Maute, Adriano Sciacovelli, and Vittorio Verda. Design of effective fins for fast pcm melting and solidification in shelland-tube latent heat thermal energy storage through topology optimization. Applied Energy, 208:210-227, 2017.
Walter A Strauss. Partial differential equations: An introduction. Wiley, 2007.

Krister Svanberg. The method of moving asymptotesa new method for structural optimization. International journal for numerical methods in engineering, 24(2): 359-373, 1987.

Fengwen Wang, Boyan Stefanov Lazarov, and Ole Sigmund. On projection methods, convergence and robust formulations in topology optimization. Structural and Multidisciplinary Optimization, 43(6):767-784, dec 2011.

Zhifeng Wang. Prospectives for china's solar thermal power technology development. Energy, 35(11):44174420, 2010.

Stephen Whitaker. Flow in porous media i: A theoretical derivation of darcy's law. Transport in porous media, 1(1):3-25, 1986.

Gil Ho Yoon. Topology optimization for stationary fluidstructure interaction problems using a new monolithic formulation. International journal for numerical methods in engineering, 82(5):591-616, 2010.

Xi Zhao, Mingdong Zhou, Ole Sigmund, and Casper Schousboe Andreasen. A poor mans approach to topology optimization of cooling channels based on a darcy flow model. International Journal of Heat and Mass Transfer, 116:1108-1123, 2018. 